\title{
The AdvancedMachines Library: Loss Models for Electric Machines
}

\author{
Anton Haumer Christian Kral Hansjörg Kapeller Thomas Bäuml Johannes V. Gragger \\ Austrian Institute of Technology \\ Giefinggasse 2, 1210 Vienna, Austria \\ anton.haumer@ait.ac.at
}

\begin{abstract}
This paper presents how losses of electric machines are modeled as an extension of the Modelica.Electrical.Machines library. The theoretical background of the different loss models is elaborated and a Modelica implementation - the AdvancedMachines Library - is presented. Additional examples demonstrate the usage of the library.
\end{abstract}

Keywords: electric machines, losses, loss models

\section{Introduction}

Especially for simulations with the goal to determine energy consumption of a system - e.g. an electric vehicle - over a given load cycle, consideration of losses as well as the variation of losses with respect to load, speed and temperature is indispensable. Since the Modelica Standard Library (MSL) Modelica.Electrical.Machines provides only basic machine models which only take copper losses caused by constant winding resistor models into account, an extension of these machine models is desired.

The different losses that have to be considered are described in [5], [6], [7]:

- Copper losses in stator and rotor windings respectively rotor cage: These losses are coupled with voltage drops; they vary with the current flowing through the winding and are temperature dependent. Though the rotor cage of an asynchronous induction machine or even the winding may be built of another conductor material like brass or aluminum, these losses are usually called "copper losses".

- Brush losses model the losses caused by the voltage drop across brushes, as needed for DC machines and slipring motors.
- Core losses in stator and rotor iron core: They vary with the quality of the used iron sheets as well as with magnetic flux and frequency of the magnetic field.

- Friction losses summarize friction at the surface of the rotor, at the bearings as well as windage losses caused by cooling fans that are mounted on the machine's shaft. They are dependent on speed.

- Stray load losses are difficult to compute and/or measure with reasonable effort [9]. Therefore their magnitude is defined in standards ([6], [7]), but no hints are given for their variation with speed. In [8] the different sources of stray load losses are explained, which allows to define the variability of these losses with respect to current and speed.

Since copper losses are temperature dependent, it is necessary to provide the actual operation temperature of the windings. This can be done by connecting a thermal ambient model which calculates the operating temperatures depending on the actual losses [2]. The simplest thermal ambient model considers constant operating temperatures, however.

Although other than copper losses are not temperature dependent, thermal connectors for all losses are included in order to provide a proper implementation which dissipates all losses, enabling a correct energy balance. They are needed for coupling a detailed thermal model to the electrical machine model.

\section{Loss Models}

\subsection{Copper Losses}

These losses are modeled by temperature dependent resistances:

$R_{\text {Operation }}=R_{\text {ref }} \cdot\left(1+\alpha_{\text {ref }} \cdot\left(T_{\text {Operation }}-T_{\text {ref }}\right)\right)$ 
where $\alpha_{\text {ref }}$ identifies the linear temperature coefficient of the specific material, with respect to the reference temperature $T_{\text {ref }}$ :

$$
\alpha_{r e f}=\frac{\alpha_{20^{\circ} \mathrm{C}}}{1+\alpha_{20^{\circ} \mathrm{C}} \cdot\left(T_{r e f}-293.15\right)}
$$

The losses are calculated by $p_{\text {loss }}=v \cdot i$ where $i$ identifies the current flowing through the resistor and $v=R_{\text {Operation }} \cdot i$ indicates the voltage drop across the component. Losses are dissipated to the component's thermal connector.

\subsection{Brush Losses}

The voltage drop $v$ across brushes is considered to be independent of the current $i$. Nevertheless, the voltage drop changes its direction according to the direction of the current flow. In order to avoid numerical problems, we have to define a linear transition around zero according to Fig. 1. The voltage drop $v$ is shown as a multiple of the nominal voltage drop $V$, whereas the parameter $I$ defines the transition range of the current $i$.

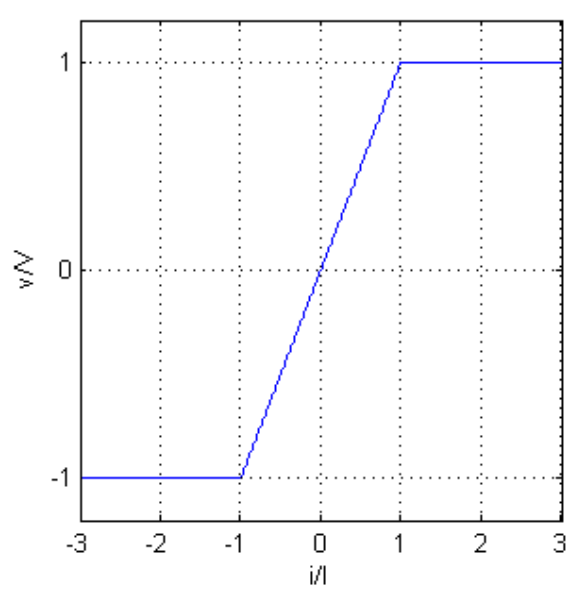

Fig. 1 Characteristic of the voltage drop across brushes

\subsection{Core Losses}

Changes of the magnetic field cause losses in the iron core which can be separated into hysteresis losses and eddy current losses. In order to avoid excessive eddy currents, the iron stack is built from sheet iron.

Assume we know the ratio of hysteresis losses $r_{H}$ with respect to the total core losses for a reference point of operation. According to [14] the core losses can be expressed depending on angular frequency, $\omega$, and flux respectively voltage, $v$ : $p=p_{\text {ref }} \cdot\left(r_{H} \cdot \frac{\omega_{r e f}}{\omega}+1-r_{H}\right) \cdot\left(\frac{v}{v_{r e f}}\right)^{2}$

Therefore core losses can be modeled as a frequency dependent conductor:

$G=\frac{p_{r e f}}{v_{r e f}^{2}} \cdot\left(r_{H} \cdot \frac{\omega_{r e f}}{\omega}+1-r_{H}\right)$

The core loss model can be connected either to the airgap model, or to the terminals.

Since the frequency of remagnetization, i.e. the velocity of the changes of the magnetic field, cannot be detected in Modelica so easily, the hysteresis losses are neglected in the first implementation $\left(r_{H}=0\right)$.

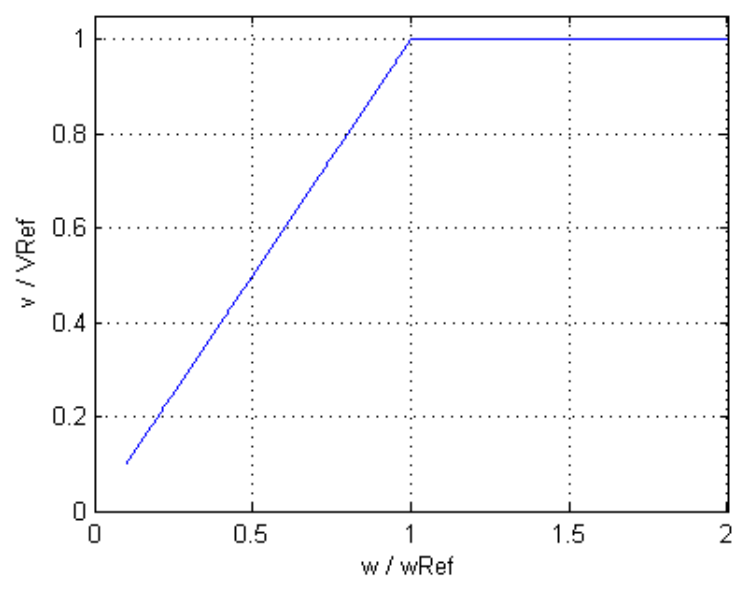

Fig. 2 Voltage versus angular velocity

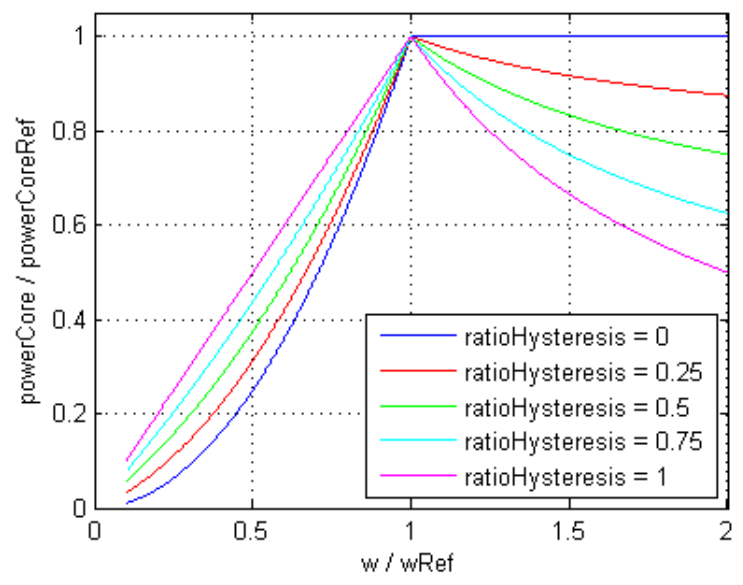

Fig. 3 Core losses versus angular velocity with parameter $r_{H}$ (ratioHysteresis).

However, we should know the influence of this simplification. Fig. 2 shows the typical dependency of voltage on angular velocity for a variable speed drive. For such a voltage/frequency relationship the characteristic of core losses dependent on angular velocity is shown in Fig. 3. In the region of constant 
flux $(w / w R e f<1)$, the core losses are underestimated, and in the region of field weakening $(w / w R e f>1)$, the core losses are overestimated.

However, determining the correct velocity of the changes of the magnet field will be subject for further investigations.

\section{$2.4 \quad$ Friction Losses}

Friction losses are caused by different phenomena:

- The rotor surface rotates relative to the surrounding medium, normally air.

- The moving parts of the bearings cannot rotate frictionless.

- Cooling fans mounted on the machine shaft require torque respectively power to drive the medium, normally air. This amount of power is called windage loss.

All friction losses depend on speed, therefore they are calculated by the following equations:

$$
\begin{aligned}
& \text { For }|\omega|>\omega_{\text {Linear }} \text { : } \\
& \tau=\operatorname{sign}(\omega) \cdot \frac{p_{\text {ref }}}{\omega_{\text {ref }}} \cdot\left|\frac{\omega}{\omega_{\text {ref }}}\right|^{\text {power }_{\omega}-1} \\
& \text { For }-\omega_{\text {Linear }} \leq \omega \leq+\omega_{\text {Linear }} \text { : } \\
& \tau=\frac{p_{\text {ref }}}{\omega_{\text {ref }}} \cdot\left(\frac{\omega_{\text {Linear }}}{\omega_{\text {ref }}}\right)^{\text {power }-1} \cdot\left(\frac{\omega}{\omega_{\text {Linear }}}\right)
\end{aligned}
$$

A linearization around zero speed is defined by $\omega_{\text {Linear }}$ for stability reasons, according to Fig. 4 .

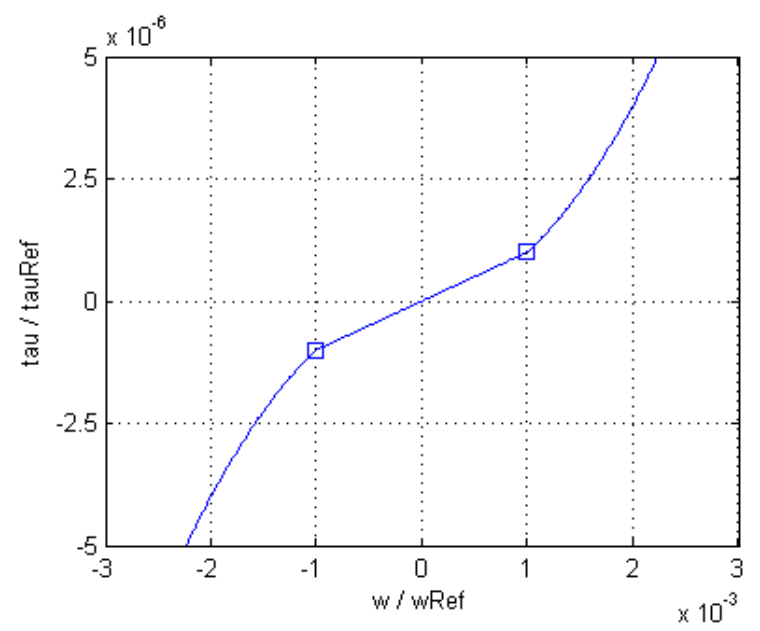

Fig. 4 Friction torque versus angular velocity

\subsection{Stray Load Losses}

Since stray load losses cannot be computed or measured with reasonable effort, their magnitude is defined in standards ([6], [7]). Unfortunately these standards deal with machines connected to a constant grid, operating at nearly constant speed. Therefore the results presented in [8] were taken as a basis to define the variability with respect to speed:

$\tau=\frac{p_{\text {ref }}}{\omega_{\text {ref }}} \cdot\left(\frac{i}{I_{\text {ref }}}\right)^{2} \cdot\left(\frac{\omega}{\omega_{\text {ref }}}\right)^{\text {power }_{\omega}-1}$

The stray load losses are defined by reference power at reference conditions $I_{r e f}$ and $\omega_{r e f}$. For induction machines $i$ respectively $I_{\text {ref }}$ designate the magnitude of the current phasor divided by $\sqrt{2}$.

The dependency of stray load losses on speed is modeled with the exponent power $_{\omega}$.

Since a voltage drop associated with the stray load losses seems to be unphysical, they are modeled as a braking torque according to [7] acting on the shaft. Again, the stray load losses $p=\tau \cdot \omega$ are dissipated to the component's thermal connector.

For stability reasons, a similar linearization as for the friction losses (6) can be implemented additionally.

\subsection{Thermal Connectors and Ambients}

In order to provide operation temperatures to copper loss models, as well as to establish a proper power balance, all loss models are provided with thermal connectors Modelica.Thermal. HeatTransfer. Interfaces. HeatPort.

Each ready-to-use machine model instantiates a machine-specific super-port, containing heat ports for all loss models which are in turn connected to that super-port. From outside, this super-port has to be connected to an ambient model, which collects all losses as well as provides all operation temperatures. This implementation allows comfortable and flexible usage.

The simplest ambient models provide constant operating temperatures, as set by the user via parameters. More sophisticated ambient models contain detailed thermal model of the corresponding machine - simulating the actual component temperatures dependent on losses and cooling conditions. Such sophisticated models are planned for future releases. 


\section{The AdvancedMachines Library}

\subsection{Structure of the Library}

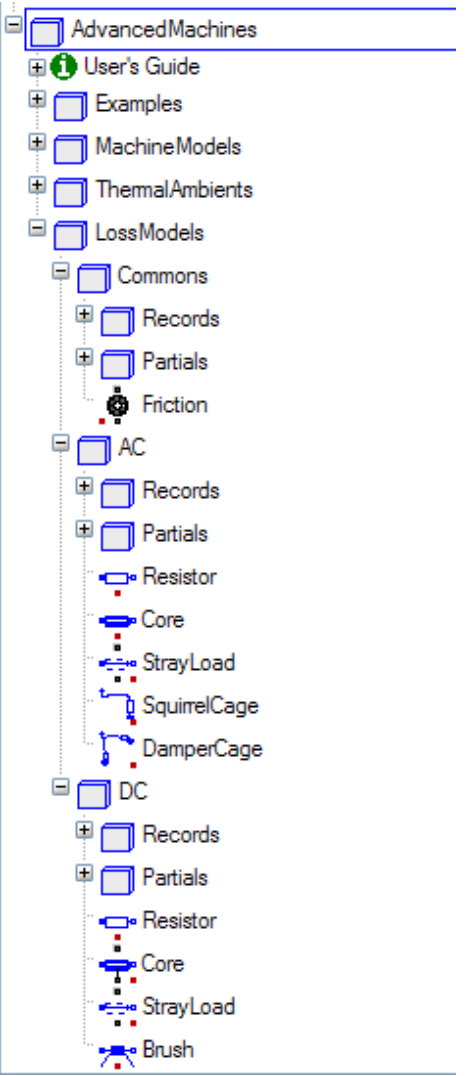

Fig. 5 Structure of the Advanced Machines Library

Besides a User's Guide and Examples, ready-to-use machine models with appropriate ambients as explained in 3.2 are provided. The loss models instantiated by the ready-to-use machine models are structured in 3 packages:

- Common to AC and DC machines

- Friction losses

o Parameter records

- Used by 3-phase AC machines

○ Temp. dependent resistor

- Core loss model

- Stray load loss model

- Symmetrical squirrel cage

- Asymmetric damper cage

- Parameter records

- Used by DC machines

○ Temp. dependent resistor
$\circ$ Core loss model
$\circ$ Stray load loss model
$\circ$ Brush loss model
$\circ$ Parameter records

\subsection{Ready-to-use Machine Models}

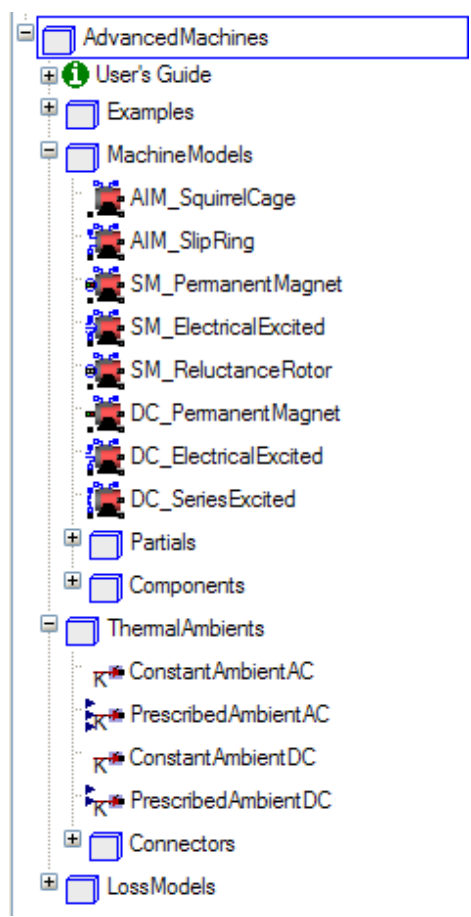

Fig. 6 Ready-to-use machine models and ambients

The AdvancedMachines library implements models for the same machine types as Modelica.Electrical.Machines, additionally taking losses into account:

- Asynchronous induction machines:

- with squirrel cage

o with slipring rotor

- Synchronous induction machines:

○ with permanent magnets

o with electrical excitation

o with reluctance rotor

- DC machines:

$\begin{array}{ll}\circ & \text { with permanent magnets } \\ \circ & \text { with electrical excitation } \\ \circ & \text { with series excitation }\end{array}$

The ambient models provide either constant or prescribed temperatures with signal inputs, both for AC induction machines and DC machines. The user has to set the appropriate machine type by means of Boolean parameters, e.g. for an asynchronous induction machine with squirrel cage:

- useRotor=true

- useRotorCage=true

- useExcitation=false

to enable the appropriate heat ports in the super-port. Future releases might split up these ambient models for more convenient usage, providing a specific ambient model for each machine type. 


\subsection{Parameterization}

In order to avoid name conflicts respectively clumsy naming for the loss parameters, like Ra_Rref or Rref Ra, all parameters needed for a specific loss model are aggregated in records:

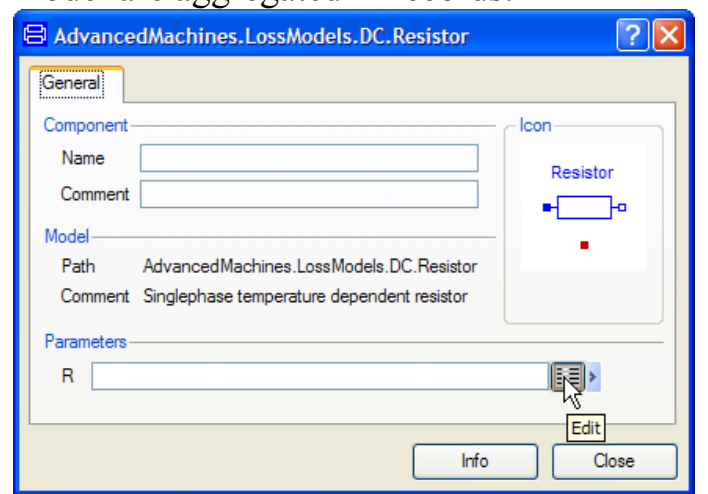

Fig. 7 Parameter record of temperature dependent resistor

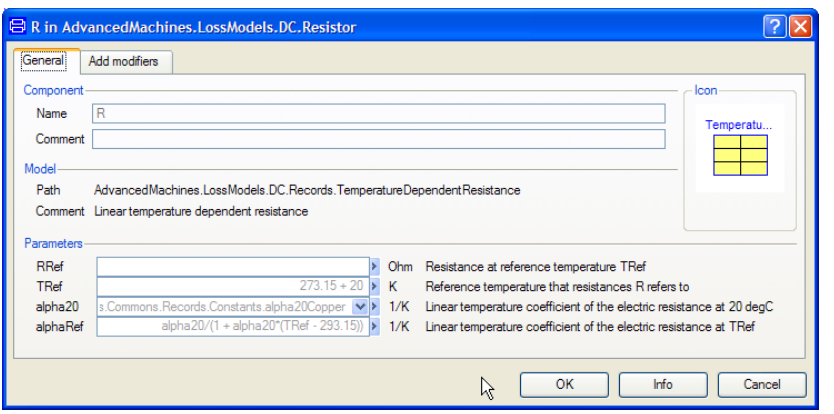

Fig. 8 Parameters of the temperature dependent resistor

These records allow to access parameters in an object oriented way, e.g. as ra.R.Rref.

Additionally, all parameters for a loss model are propagated by a single propagation of the appropriate parameter record, which allows convenient exchange and testing of different parameter settings.

The parameters of many loss models (FrictionLosses, CoreLosses, StrayLoadLosses) require to specify a reference value for the losses and corresponding reference conditions (like reference speed).

In many cases the user knows these values from manufacturer data or from test protocols, but wants to specify consistent parameter sets [12]. Consistent parameter sets means that the specified reference losses are dissipated exactly in the reference point of operation. Unfortunately, the specification of the reference point of operation (like nominal load) might be incomplete, e.g. with unknown voltage at the core loss model.

To help the user to define consistent parameter sets, a future release of the library will provide a parameter record for each ready-to-use machine model, calculating the missing reference values initially.

\section{Simulation Examples}

\subsection{Permanent Magnet Machine}

This example investigates the impact of losses on the behavior of a DC permanent magnet machine, based on the default machine data used in the MSL:

\begin{tabular}{|lrrr|}
\multicolumn{1}{l}{} & $\begin{array}{r}\text { Standard } \\
\text { Machine }\end{array}$ & $\begin{array}{r}\text { Advanced } \\
\text { Machine }\end{array}$ \\
\hline Armature voltage & 100 & 100 & $\mathrm{~V}$ \\
Armature current & 100 & 100 & $\mathrm{~A}$ \\
Nominal speed & 1425.0 & 1417.5 & $\mathrm{rpm}$ \\
Inner voltage & 95 & 94.5 & $\mathrm{~V}$ \\
\hline Armature resistance & 0.05000 & 0.03864 & $\Omega$ \\
Temperature coefficient & $\mathrm{n} / \mathrm{a}$ & 0.00392 & $1 / \mathrm{K}$ \\
Reference temperature & 95 & 20 & ${ }^{\circ} \mathrm{C}$ \\
Operation temperature & $\mathrm{n} / \mathrm{a}$ & 95 & ${ }^{\circ} \mathrm{C}$ \\
\hline Brush Voltage drop & 0 & 0.5 & $\mathrm{~V}$ \\
Linear transition current & $\mathrm{n} / \mathrm{a}$ & 1 & $\mathrm{~A}$ \\
\hline Core Losses & 0 & 200 & $\mathrm{~W}$ \\
Reference voltage & $\mathrm{n} / \mathrm{a}$ & 94.5 & $\mathrm{~V}$ \\
Reference speed & $\mathrm{n} / \mathrm{a}$ & 1417.5 & $\mathrm{rpm}$ \\
\hline Stray Load Losses & 0 & 50 & $\mathrm{~W}$ \\
Reference current & $\mathrm{n} / \mathrm{a}$ & 100 & $\mathrm{~A}$ \\
Reference speed & $\mathrm{n} / \mathrm{a}$ & 1417.5 & $\mathrm{rpm}$ \\
\hline Friction Losses & 0 & 100 & $\mathrm{~W}$ \\
Reference speed & $\mathrm{n} / \mathrm{a}$ & 1417.5 & $\mathrm{rpm}$ \\
\hline Electrical Input & 6000 & $10,000.00$ & $\mathrm{~W}$ \\
Armature Losses & 500.00 & 500.00 & $\mathrm{~W}$ \\
Brush Losses & 0.00 & 50.00 & $\mathrm{~W}$ \\
Core Losses & 0.00 & 200.00 & $\mathrm{~W}$ \\
Stray Load Losses & 0.00 & 50.00 & $\mathrm{~W}$ \\
Friction Losses & 0.00 & 100.00 & $\mathrm{~W}$ \\
Mechanical Ouput & $9,500.00$ & $9,100.00$ & $\mathrm{~W}$ \\
Nominal Torque & 63.66 & 61.30 & $\mathrm{Nm}$ \\
\hline
\end{tabular}

Table 1 Parameters of both DC PM machines

The inner voltage at the airgap can be calculated as:

$V_{i}=V_{a}-V_{\text {Brush }}-R_{\text {a,operation }} \cdot I_{a}=k \cdot \omega$

The magnet design of both models is considered to be the same. Since the inner voltage $V_{i}$ of the AdvancedMachine is lower than that of the StandardMachine due to the brush voltage drop, the nominal speed of the AdvancedMachine has to be lower than that of the StandardMachine. Note that the nominal torque of the AdvancedMachine is lower than that of the StandardMachine at the same electrical input, due to the losses.

Both machines are started on voltage ramp (Fig. 9) with a duration of $0.8 \mathrm{~s}$, starting at $0.2 \mathrm{~s}$. At $\mathrm{t}=1.5 \mathrm{~s}$ a torque step (respective nominal torque for each model) is applied.

The final stationary resulting speed (Fig. 10) meets the values of Table 1. Both armature currents shown 
in Fig. 11 reach $100 \mathrm{~A}$ according to Table 1, but differences can bee seen during transient operation.

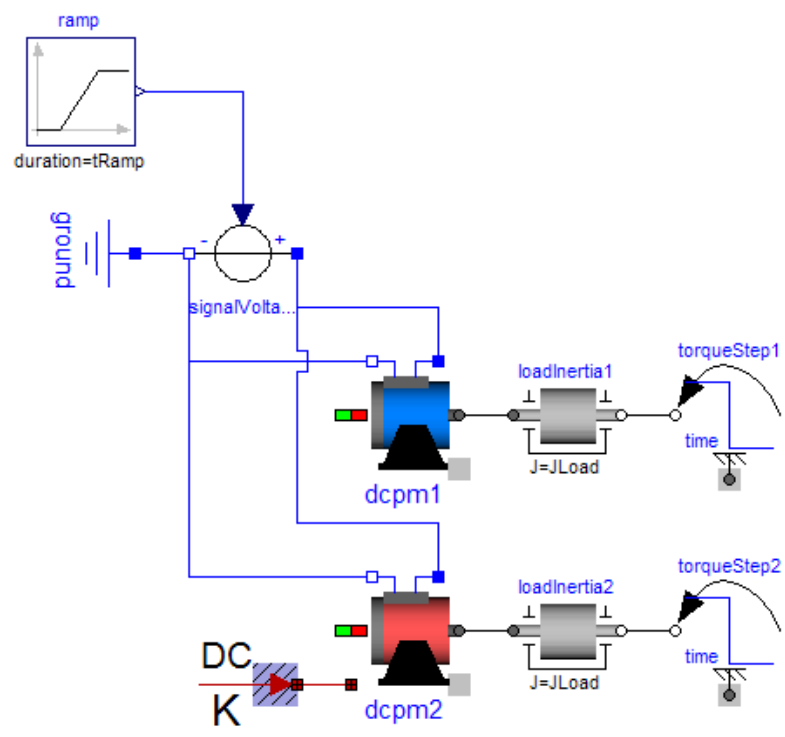

Fig. 9 Starting both DC permanent magnet machines

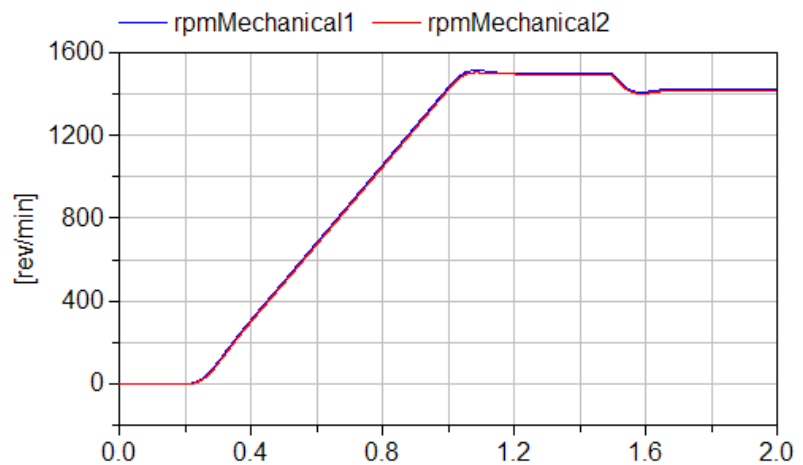

Fig. 10 Comparing speed of both machines

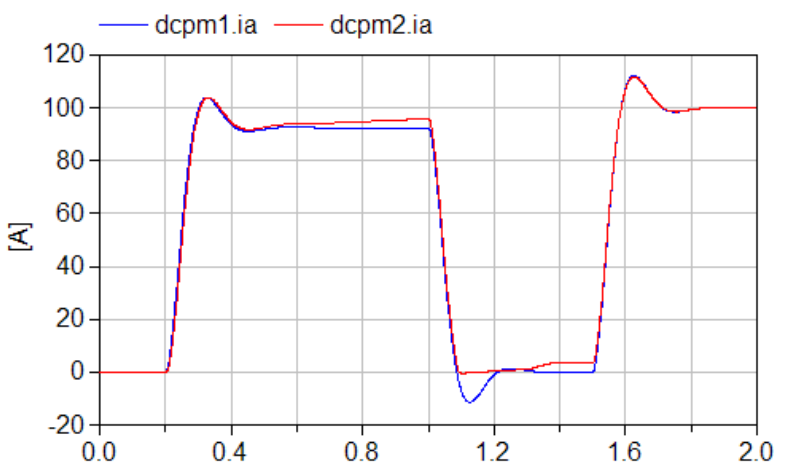

Fig. 11 Comparing armature currents of both machines

\subsection{Asynchronous Induction Machine with Squirrel Cage}

In order to validate the loss models, an asynchronous induction machine with squirrel cage (AIMC) is simulated for different partial loads. The results are compared with measurements of a $18.5 \mathrm{~kW}$ 4-pole standard motor (Table 2). The motor is of totally en- closed fan cooled design (Fig. 12), the rotor cage is made of aluminum (Fig. 13).

\begin{tabular}{|lrc|}
\hline Nominal output & 18,500 & $\mathrm{~W}$ \\
Nominal voltage & 400 & $\mathrm{~V}$ \\
Connection & delta & \\
Nominal freuqency & 50 & $\mathrm{~Hz}$ \\
\hline
\end{tabular}

Table 2 Nominal parameters of the AIMC

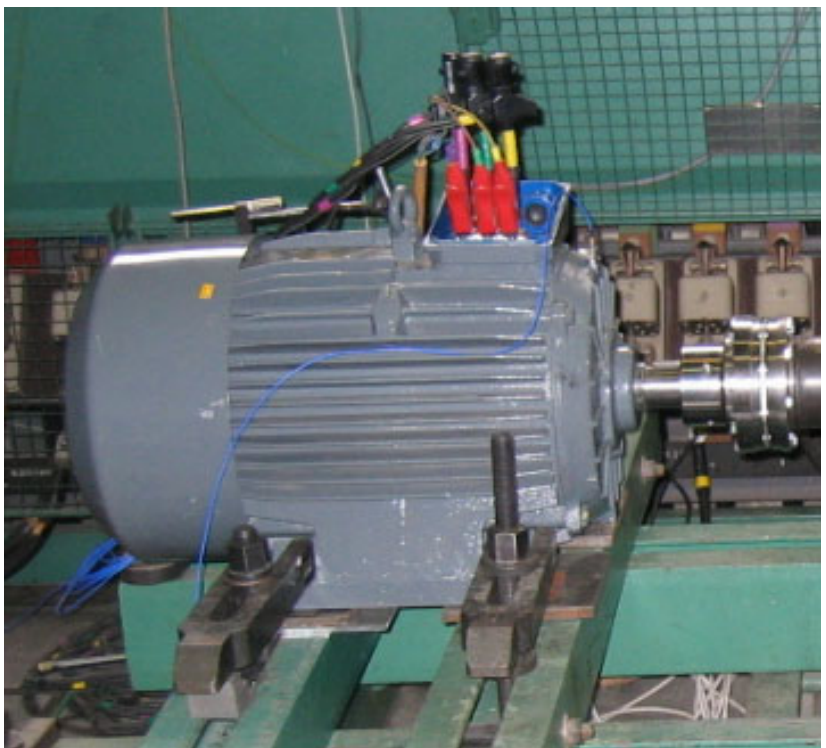

Fig. 12 The AIMC at the test bench

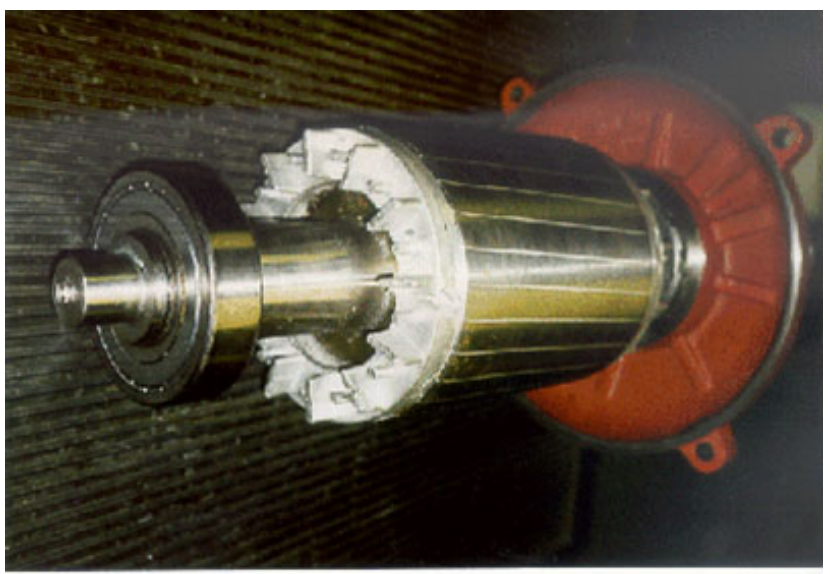

Fig. 13 Die cast aluminium rotor of the AIMC

\begin{tabular}{|lrr|}
\hline Stator resistance / phase & 0.560 & $\Omega$ \\
Temperature coefficient & 0.00392 & $1 / \mathrm{K}$ \\
Reference temperature & 20 & ${ }^{\circ} \mathrm{C}$ \\
Operation temperature & 90 & ${ }^{\circ} \mathrm{C}$ \\
\hline Stator leakage reactance & 1.520 & $\Omega$ \\
Main reactance & 66.400 & $\Omega$ \\
Rotor leakage reactance & 2.310 & $\Omega$ \\
\hline Rotor resistance / phase & 0.420 & $\Omega$ \\
Temperature coefficient & 0.00400 & $1 / \mathrm{K}$ \\
Reference temperature & 20 & ${ }^{\circ} \mathrm{C}$ \\
Operation temperature & 90 & ${ }^{\circ} \mathrm{C}$ \\
\hline
\end{tabular}

Table 3 Impedances of the AIMC

rotor impedances with respect to the stator 
The impedances (Table 3) were taken from the results of a conventional design program, the losses (Table 4) were taken from a type test protocol.

The voltage drop across the core loss conductance for nominal operation $\left(V_{\text {core }}=375.7 \mathrm{~V}\right)$ was iterated with the model shown in Fig. 14, which was used to obtain the results, too.

\begin{tabular}{|lrr|}
\hline Stator current & 32.85 & A \\
Power factor & 0.898 & \\
Speed & 1462.5 & $\mathrm{rpm}$ \\
\hline Electrical input & $20,443.95$ & W \\
Stator copper losses & 770.13 & W \\
Core losses & 410.00 & W \\
Rotor copper losses & 481.60 & W \\
Stray load losses & 102.22 & W \\
Friction losses & 180.00 & W \\
Mechanical output & $18,500.00$ & W \\
\hline Efficiency & $90.49 \%$ & \\
Nominal Torque & 120.79 & $\mathrm{Nm}$ \\
\hline
\end{tabular}

Table 4 Nominal operation of the AIMC

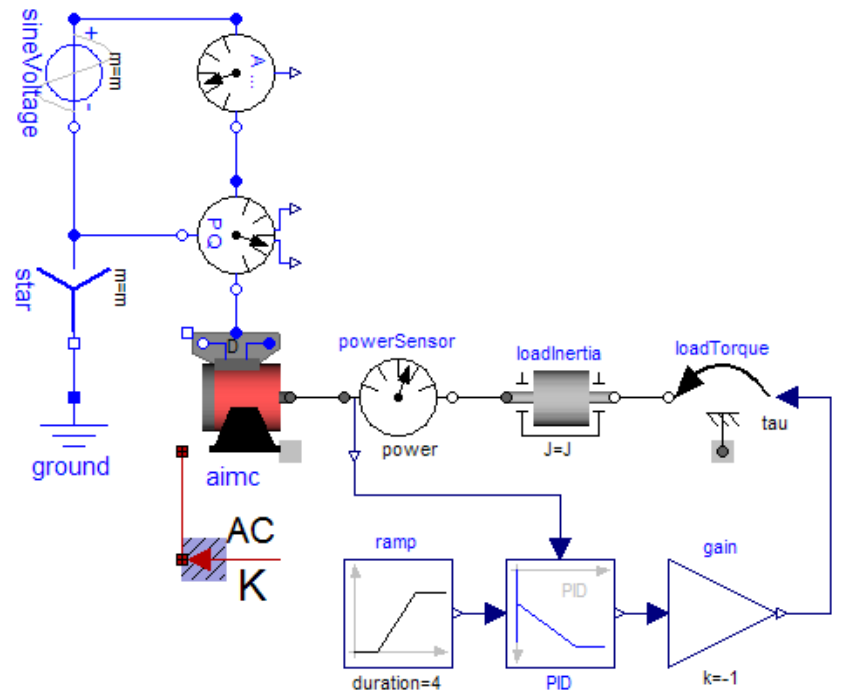

Fig. 14 Simulation model of the AIMC

The asynchronous induction machine is started at nominal speed and fed from a constant grid with nominal voltage and frequency. The load torque is controlled to achieve the desired mechanical power. After the initial transients have vanished, the set point for the mechanical power is raised with a very slow ramp to achieve quasi-stationary operation.

The comparison of measurement and simulation results (Fig. 15 - Fig. 17) shows very good coincidence which proofs the validity of the presented load models. Remaining differences can be explained due to measurement uncertainty, the fact that the calculated impedances used for parameterization do not match that of the real machine exactly which gives rise to deviations mainly in reactive power and last but not least the fact that the test protocol calculates the losses slightly different from the presented loss models.

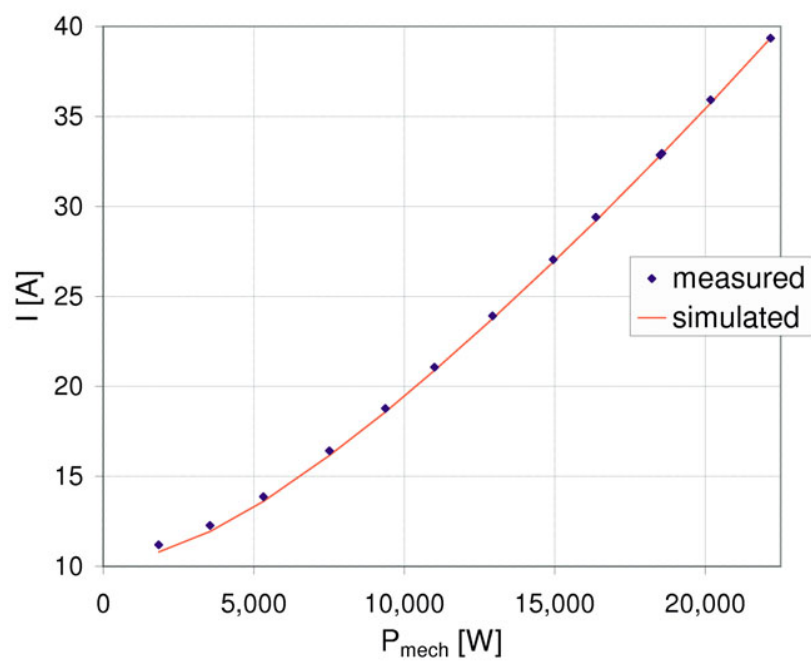

Fig. 15 Current of the AIMC,

simulation results compared with measurements

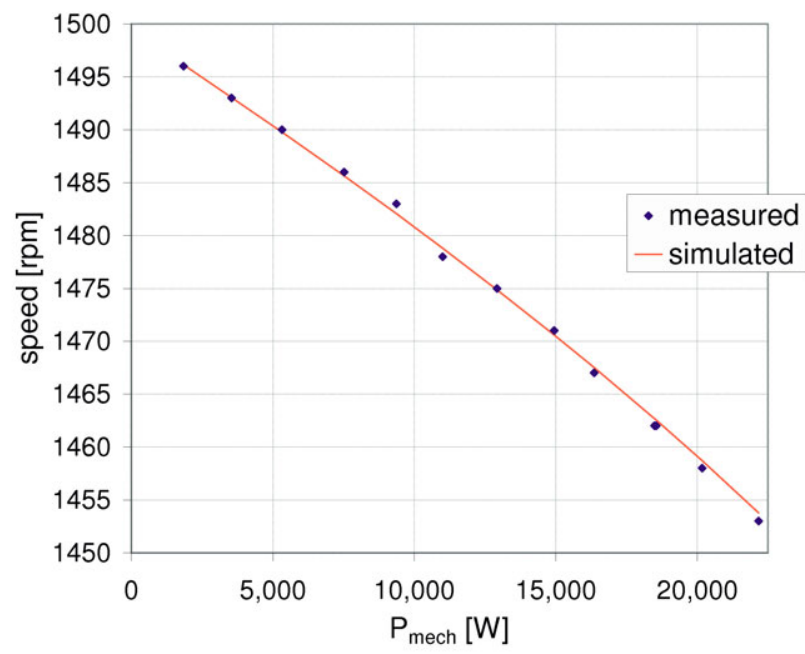

Fig. 16 Speed of the AIMC,

simulation results compared with measurements

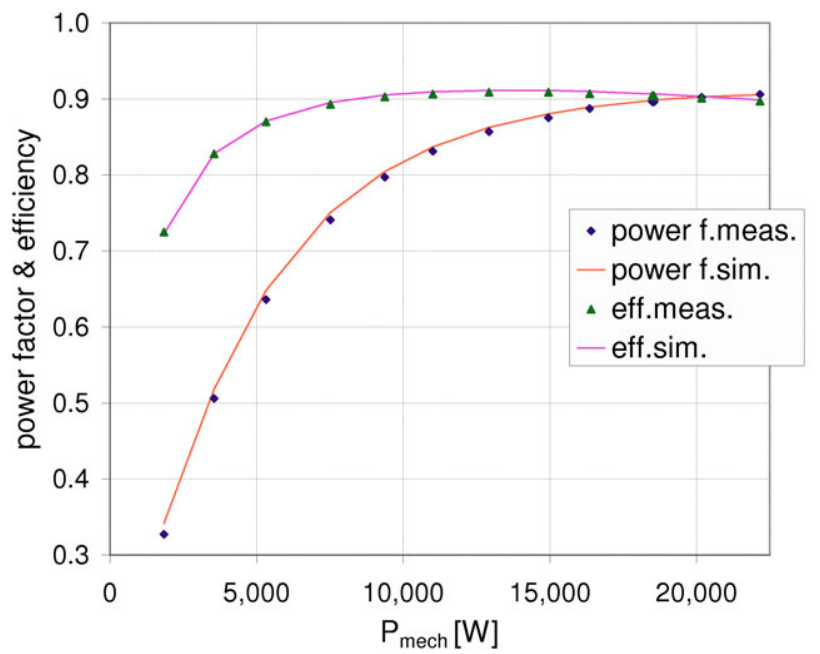

Fig. 17 Power factor and efficiency of the AIMC, simulation results compared with measurements 


\section{Conclusions and Outlook}

The design of a Modelica library for electric machines with detailed loss models has been presented. The structure of the different loss models - copper losses, brush losses, core losses, friction and stray load losses - has been discussed in detail. Furthermore, the usage of the library has been presented with two examples, one of them providing a comparison between simulated and measured losses.

It is planned to release the library as a supplement for the SmartElectricDrives Library [4]. In this context also quasi-stationary models are of interest. As soon as it is possible to achieve a stable implementation of quasi-stationary machine models - which depends on the implementation of complex numbers in Modelica - as described in [3], the loss models will be adapted for the quasi-stationary machine models.

In future releases simple thermal models of electric machines will be offered. Additionally, the impact of coupling electric and thermal models will be investigated. Since the time constants of the electric and the thermal part are different, co-simulation could be considered to improve simulation performance.

\section{References}

[1] C. Kral, A. Haumer, Modelica libraries for dc machines, three phase and polyphase machines. $4^{\text {th }}$ International Modelica Conference 2005, Hamburg, Germany

[2] C. Kral, A. Haumer, M. Plainer, Simulation of a thermal model of a surface cooled squirrel cage induction machine by means of the SimpleFlow-library. $4^{\text {th }}$ International Modelica Conference 2005, Hamburg, Germany

[3] A. Haumer, C. Kral, J. Gragger, H. Kapeller, Quasi-Stationary Modeling and Simulation of Electrical Circuits using Complex Phasors. $6^{\text {th }}$ International Modelica Conference 2008, Bielefeld, Germany

[4] J. Gragger, H. Giuliani, C. Kral, T. Bäuml, H. Kapeller, F. Pirker, The SmartElectricDrives Library - Powerful Models for Fast Simulation of Electric Drives. $5^{\text {th }}$ International Modelica Conference 2006, Vienna, Austria

[5] H. Kleinrath, Grundlagen elektrischer Maschinen, Akademische Verlagsgesellschaft, Wiesbaden, 1975
[6] Standard EN 60034-2, Verfahren zur Bestimmung der Verluste und des Wirkungsgrades von drehenden elektrischen Maschinen aus Prüfungen

[7] IEEE Standard 112, IEEE standard test procedure for polyphase induction motors and generators

[8] W. Lang, Über die Bemessung verlustarmer Asynchronmotoren mit Käfigläufer für Pulsumrichterspeisung, Doctoral Thesis, Technical University of Vienna, 1984

[9] M. Aoulkadi, A. Binder, When Loads Stray: Evaluation of Different Methods to Determine Stray Load Losses in Induction Machines, IEEE Industrial Electronics Magazine, 2008, Vol. 2, No. 1, p. 21-30

[10] H. Spaeth, Elektrische Maschinen - Eine Einführung in die Theorie des Betriebsverhaltens, Springer-Verlag, Berlin-HeidelbergNew York, 1973

[11] K.P. Kovac, I. Racz, Transiente Vorgänge in Wechselstrommaschinen, Band I, Verlag der Ungarischen Akademie der Wissenschaften, Budapest 1959

[12] C. Kral, A. Haumer, Consistent Equivalent Circuit Parameters of Induction Motors for the Calculation of Partial Load Efficiencies. IEEE International Symposium on Industrial Electronics, ISIE, Cambridge, United Kingdom, 2008

[13] M. Schelch, Motor-Pre-Calculator, Diploma Thesis, Technikum Wien, Vienna, 2005

[14] D. Lin, P. Zhou, W. Fu, Z. Badics, Z. Cendes, A dynamic core loss model for soft ferromagnetic and power ferrite materials in transient finite element analysis, Conference Proceedings COMPUMAG, 2003 